\title{
Design of nuclear radiation detector for stack exhaust of plasma melting system
}

\author{
Qingxin Lei ${ }^{1 *}$, Chenyu Shan ${ }^{1}$, Chunyu Liu ${ }^{1}$, Xiajie Liu ${ }^{1}$, Wenzhang Xie ${ }^{1}$ \\ ${ }^{1}$ China Nuclear Power Technology Research Institute, Shenzhen 518031, China
}

\begin{abstract}
The Plasma melting technology can reduce the low and medium level radioactive waste to a minimum. The emission of high-temperature radioactive aerosol generated in the process of treatment needs to be monitored. After treatment with a high efficiency filter and meeting national standards for gaseous effluents, it will be allowed to be discharged into the atmosphere. In order to achieve stable and reliable radiation monitoring, through the research on the radionuclide analysis of stack exhaust and the design of detector selection, the combined design of plastic scintillator \& NaI scintillator is adopted. At the same time, the real-time and reliable monitoring of radioactive aerosols in stack gases under high temperature environment is also solved.
\end{abstract}

\section{Introduction}

A large amount of radioactive wastes will be generated during the operation of nuclear power plants. Solving the practical disposal problem is not only related to the improvement of environmental protection concept, but also the requirement of improving the economic efficiency of nuclear power plants. As the most advanced solid waste treatment technology recognized in the world, plasma melt technology has been dubbed as the "terminator of solid waste". The radioactive waste can be minimized by using the plasma melt reduction process. But in the use of plasma melting technology of dealing with the low-level radioactive waste, some waste gas will be produced in the process, including dioxins, NOx, SOx, and other conventional pollutants such as fly ash, and the volatile radioactive nuclides, the pollutants and radionuclides can be effectively removed through the stack high-efficiency air filter ${ }^{[1]}$. According to the requirements of GB62492011 "nuclear power plant environment radiation protection regulations", when the gaseous effluent is discharged through the stack, the necessary continuous monitoring must be carried out. Therefore, the continuous monitoring device of radioactivity must be set up for the stack exhaust of the plasma melting system.

\section{Radioactive Aerosols}

Plasma incineration system is used to treat low and medium level radioactive waste from nuclear power plant. The stack gas contains radioactive aerosols, which are composed of solid or liquid particles (or both) suspended in the gas. The particle diameter range is about $10^{-3}-10^{3} \mu$ $\mathrm{m}$. Radon and its daughter products in the air are radioactive, and they become radioactive aerosols that are absorbed by atmospheric suspended substances. It has the characteristic of high ionization effect and low concentration, and is also the main threat to human irradiation. From the point of view of radiation protection, effective and accurate continuous monitoring of radioactive aerosol concentration is to protect the health of personnel from the risk of excessive radiation exposure.

Because of the existence of radon, thorium and their decay daughters in the air, it is very difficult to monitor the aerosol in stack gas in real-time and quickly. In order to make it easy to monitor the activity concentration of radioactive aerosol when it is very low, it is usually not measured directly, and then let a large amount of sampling gas pass through the filter paper, the radioactive aerosol is deposited on the surface of the filter paper, the radioactive activity concentration of aerosol in the air (e.g. $\left.\mathrm{Bq} / \mathrm{m}^{3}\right)$ is calculated by the nuclear radiation detector ${ }^{[2]}$.

\section{Design of Nuclear Radiation Detector}

\subsection{Source Term Analysis}

According to the operation experience of low and medium level radioactive wastes in nuclear power plants, the types of wastes treated by plasma melt system include cotton products, absorbent paper, PE plastics and PVC plastics, which the cotton goods are the majority. According to the material ratio of $80 \%$ cotton products $+15 \%$ PE plastics + $5 \%$ PVC plastics, the composition of the four materials was analysed. The isotope tracer technology was used to trace the radionuclides in the gas finally entering the stack, and the specific activity of radionuclides in the gas was calculated, as shown in Figure 1. 


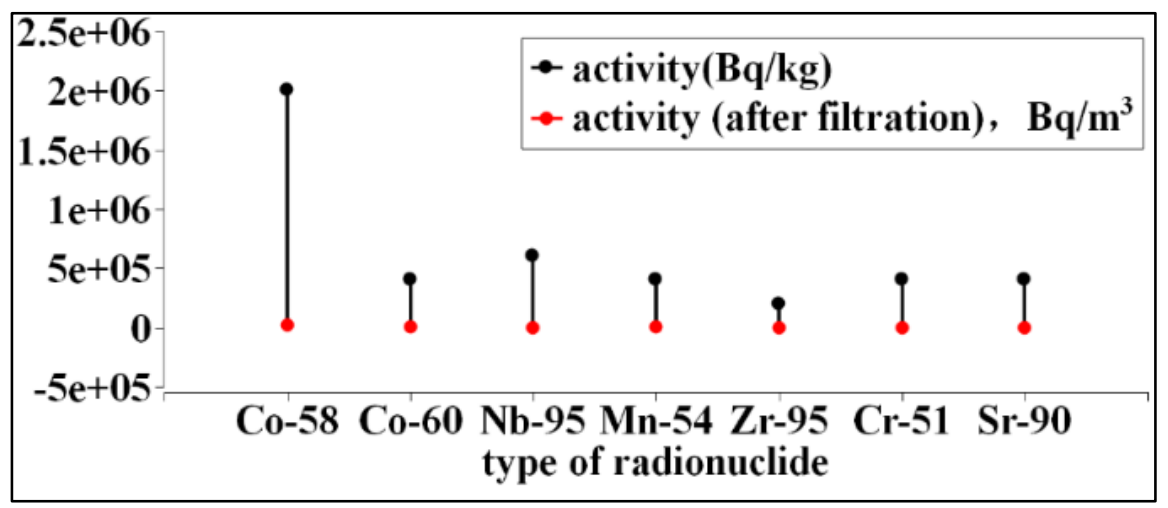

Fig.1 Radioactivity of radionuclides before and after filtration

As it can be seen from Figure 1, after the reduction treatment of low-level radioactive waste, most of the radionuclides discharged are directly removed by the high-efficiency air filter. When the filter is slightly damaged or may fail, the aerosol in the stack exhaust will be detected by the nuclear radiation detector, and then remind the staff to replace the filter or take further safety measures.

Table 1 Radionuclide characteristics of radioactive aerosol from stack exhaust

\begin{tabular}{|c|c|c|c|c|c|c|}
\hline \multirow[b]{2}{*}{ Nuclide } & \multirow[b]{2}{*}{ The Half-life } & \multicolumn{3}{|c|}{ Major $\beta$ Rays } & \multicolumn{2}{|c|}{ Major $\gamma$ Rays } \\
\hline & & $\begin{array}{c}\text { Maximum } \\
\text { Energy }(\mathrm{KeV})\end{array}$ & $\begin{array}{l}\text { Average } \\
\text { Energy } \\
(\mathrm{KeV}) \\
\end{array}$ & $\begin{array}{c}\text { The Intensity } \\
(\%)\end{array}$ & $\begin{array}{c}\text { Energy, } \\
\mathrm{KeV}\end{array}$ & $\begin{array}{c}\text { The Intensity } \\
(\%)\end{array}$ \\
\hline Co-58 & $70.86 \mathrm{~d}$ & 475 & 201.2 & 14.93 & 810.8 & 99.43 \\
\hline Co- 60 & $5.27 \mathrm{a}$ & 317.9 & 95.8 & 100 & $\begin{array}{l}1173.2 \\
1332.5\end{array}$ & $\begin{array}{l}100 \\
100\end{array}$ \\
\hline $\mathrm{Nb}-95$ & $35.06 \mathrm{~d}$ & 159.8 & 43.4 & 99.97 & 765.8 & 99.80 \\
\hline $\mathrm{Mn}-54$ & $312.7 \mathrm{~d}$ & 693.6 & 290.1 & - & 834.8 & 99.98 \\
\hline Zr-95 & $64.02 \mathrm{~d}$ & $\begin{array}{l}366.4 \\
398.9\end{array}$ & $\begin{array}{l}109.3 \\
120.4\end{array}$ & $\begin{array}{l}55.40 \\
43.70\end{array}$ & $\begin{array}{l}724.2 \\
756.7\end{array}$ & $\begin{array}{l}43.66 \\
55.35\end{array}$ \\
\hline Cr-51 & $27.70 \mathrm{~d}$ & -- & -- & -- & $\begin{array}{l}0.47 \\
4.38\end{array}$ & $\begin{array}{c}144.68 \\
66.89\end{array}$ \\
\hline Sr-90 & $28.60 \mathrm{a}$ & 546 & 195.8 & 100 & -- & -- \\
\hline$Y-90$ & $64 \mathrm{~h}$ & 2280 & 933 & 99.99 & -- & -- \\
\hline C-14 & $5700 \mathrm{a}$ & 156 & 50 & 100 & -- & -- \\
\hline Note: “"-- & eans no; & & & & & \\
\hline
\end{tabular}

For the characteristics of source term distribution in the stack gas of plasma melt system, it is necessary to detect all nuclides accurately through monitoring $\beta$ rays and $\gamma$ rays together. Because of the poor penetration ability of $\beta$ - ray, online sampling monitoring method is generally used.

\subsection{Detector Selection}

For the on-line continuous monitoring of radioactive aerosols, Passivated Implanted Planar Silicon (PIPS) semiconductor detectors are commonly used in nuclear power plants (figure 2). In view of the radiation emitted by aerosol nuclide decay accumulated on filter paper, detector 1 can measure $\alpha, \beta$ and $\gamma$, and detector 2 can measure $\gamma$. Through special anti-coincidence circuit, $\gamma$ background is deducted to achieve the accurate measurement of $\beta$ aerosol.
The type of Radioactive nuclide determines the design of the nuclear radiation detector, based on the characteristics of the nuclide in table 1 , both $\beta$ radionuclides and $\gamma$ radionuclides exist in stack gases, some of radionuclide (e.g.Sr-90) is only by detecting $\beta$ rays to monitor, and some of the radionuclide (e.g.Cr-51) can be monitored through the detection of $\gamma$ rays. 
to be no more than $60^{\circ} \mathrm{C}$. When the gas temperature reaches $80{ }^{\circ} \mathrm{C}$, the leakage current of the detector will be doubled, and the background noise will almost submerge the normal measurement data, resulting in poor detection performance and unable to measure accurately ${ }^{[3]}$.

According to the fact that the nuclides in stack gas measurement are mainly $\beta$ particles, the design scheme of scintillator combination detector (plastic scintillator $+\mathrm{NaI}$ scintillator) which is relatively mature in the industry and relatively low cost is adopted. The plastic scintillator is used in the front-end to measure the aerosol total $\beta$ level and cascade $\gamma$ emission level, and the NaI scintillator is used as the detection component at the back-end to remove the $\gamma$ rays from the plastic scintillator background count.

\section{3 detector design}

The detector adopts plastic scintillator $+\mathrm{NaI}$ scintillator combination mode, which can simultaneously measure $\beta$ count and $\gamma$ spectrum. By controlling the thickness of plastic scintillator, the response to $\gamma$ photons can be greatly reduced and the crosstalk caused by $\gamma$ photons can be reduced ${ }^{[4]}$

1) Measurement of $\beta$ particles (plastic scintillator)

For the measurement of $\beta$ particle, it has the characteristics of fast response and high detection efficiency, and outputting the counting rate in real-time. Combined with detection efficiency, the radioactive activity concentration of $\beta$ particle can be calculated.

2) Measurement of $\gamma$ photons (NaI scintillator)

It has a high photon yield. At the same time, when the high temperature is $160{ }^{\circ} \mathrm{C}$, the photon yield only decreases to 0.9 times at normal temperature, which is suitable for working in high temperature environment (see Fig. 3). It can obtain energy spectrum data, identify radionuclides and output radioactivity concentration.

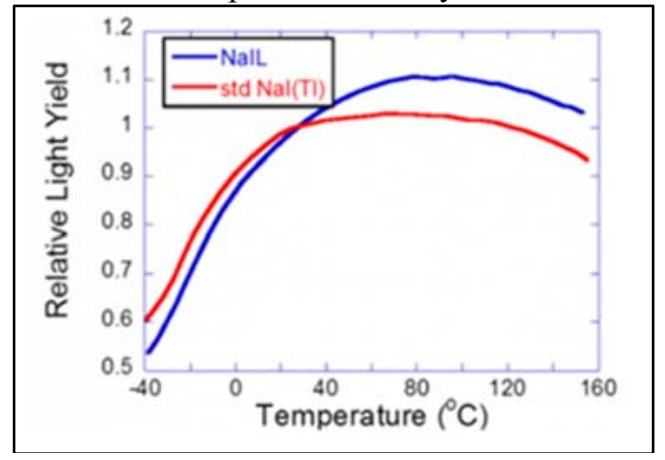

Fig. 3 The relation curve between photon yield of $\mathrm{NaI}$ and temperature

\subsubsection{Detection Efficiency of $\beta$ Particles in Plastic Scintillator}

The $\beta$ particles in the detector design are mainly measured by plastic scintillator, that is organic scintillators used for the detection of $\alpha, \beta, \gamma$, fast neutrons, protons, cosmic rays and fission fragments etc. It is easy to make large transparent body and easy to process into various shapes. It has the advantages of no deliquescence, stable performance, radiation resistance, short scintillation attenuation time and low price. The penetration of $\beta$ particles in plastic scintillators is much lower than that of $\gamma$ photons, so the thinner plastic scintillator is adopted to reduce the noise caused by $\gamma$ photons. In this design, $0.25 \mathrm{~mm}$ thick plastic scintillator is selected, which is also the thinnest size in China at present, and it has passed the performance test at $170^{\circ} \mathrm{C}$.

It can be seen from table 1 that the maximum energy of $\beta$ particles is $2.28 \mathrm{Mev}$. Since the $\beta$ radiation is a continuous spectrum, its energy is distributed within the range of $0 \sim$ maximum energy, and the average energy of electronic energy spectrum is usually taken as reference. Analyzing the radiation of each nuclide in the table 1, the average electron energy of $90 \mathrm{Y}$ is $933 \mathrm{kev}, 14 \mathrm{C}$ is $50 \mathrm{kev}$, the average electron energy of other nuclides is between 100 and $200 \mathrm{keV}$. Fig. 4 shows the detection efficiency curve of $\beta$ particles simulated by Monte Carlo method, for $\beta$ particles with $100 \sim 200 \mathrm{keV}$, the detection efficiency is about $40 \%$. With the increase of electron energy, the detection efficiency decreases slightly, dropping to $37 \%$ when the electron energy reaches $1000 \mathrm{keV}$, and to $35 \%$ when the electron energy reaches $2500 \mathrm{keV}$.

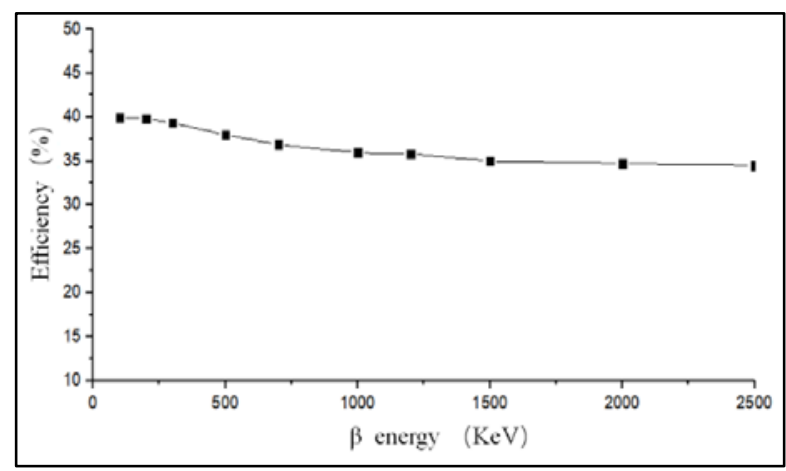

Fig. 4 Detection efficiency curve of $\beta$ particle on a plastic scintillator

\subsubsection{Response of y Photons in Plastic Scintillators}

$\gamma$ photons can also generate signals in plastic scintillators, and their signal waveforms are so similar to those of $\beta$ particles, so they cannot be distinguished from each other. In order to reduce the interference caused by $\gamma$ photons, ultra-thin plastic scintillation slices were selected in order to reduce the influence of $\gamma$ photons.

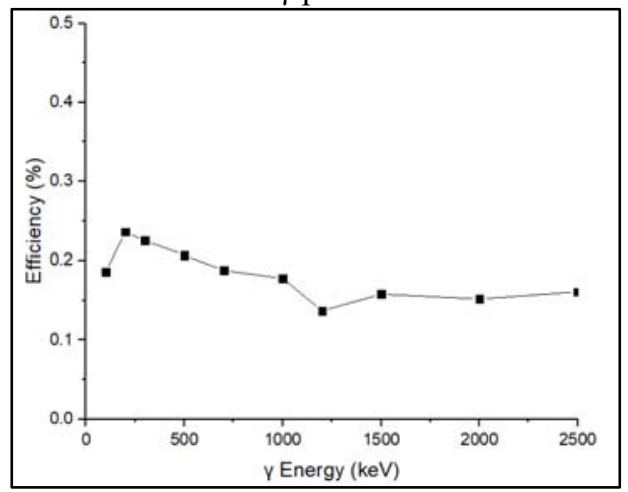

Fig.5 Response efficiency curve of $\gamma$ photon on plastic scintillator 
Fig. 5 shows the simulated response of different energy $\gamma$ photons on the plastic scintillator ${ }^{[5]}$, the absolute response efficiency is about $0.2 \%$. With the absolute detection efficiency of $40 \%$ for $\beta$ particles, when the number of $\gamma$ photons has the same as that of $\beta$ particles, the interference effect of $\gamma$ photons on $\beta$ particles is about $0.5 \%$. If the $\gamma$ photon background in the external environment is higher, the $\gamma$ cross-talk will also be increased, which is very related to the real measurement environment. The detector needs to be calibrated onsite, and the response ratio of $\mathrm{NaI}$ scintillators and plastic scintillators to photons was obtained to calculate the actual response data of plastic scintillators to $\gamma$ photons, thus improving the measurement accuracy of $\beta$ particles.

\subsubsection{Simulation of Quartz Glass Thickness}

For high energy $\beta$ particles, it cannot be completely blocked by $0.25 \mathrm{~mm}$ plastic scintillator, so a thickness of quartz glass is added between the plastic scintillation and the $\mathrm{NaI}$ to absorb the $\beta$ particles energy, and prevent the particles from entering the $\mathrm{NaI}$ crystal, $\mathrm{NaI}$ scintillator is made of $\varphi 50 * 36 \mathrm{~mm}$, because it is easy to deliquescence, it also needs to be packaged. The upper surface is sealed with $5 \mathrm{~mm}$ thick quartz glass, the lower surface is sealed with $1 \mathrm{~mm}$ thick quartz glass, and the side is packaged with $1.5 \mathrm{~mm}$ thick aluminium shell.

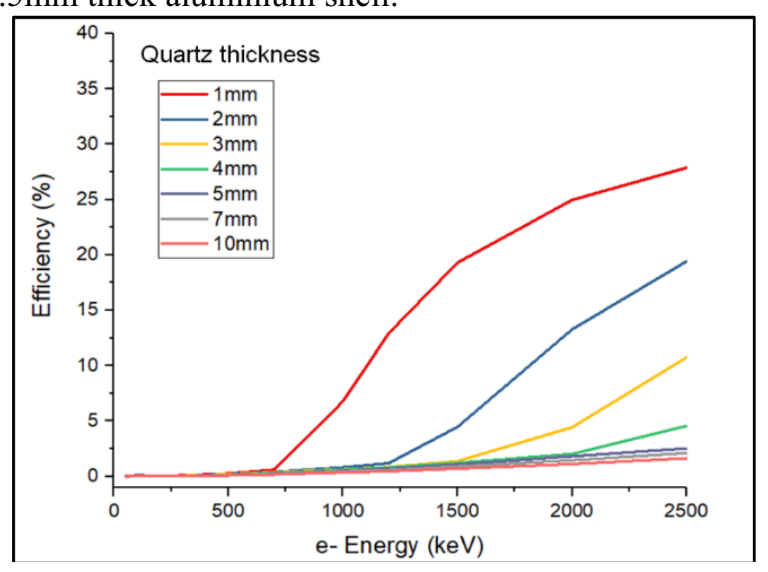

Fig.6 Response curves of $\beta$ particles on different quartz glass thickness

As shown in the figure $6, \mathrm{NaI}$ response curves to different $\beta$ particle energies were simulated under the condition of different thickness quartz glass by using $0.25 \mathrm{~mm}$ thick plastic scintillation. The results showed that the response efficiency of $\mathrm{NaI}$ to $2.5 \mathrm{MeV} \beta$ particles was less than $1 \%$, when the thickness was $5 \mathrm{~mm}$, it didn't go down to 0 . Because of the high-energy electron pair effect, the positive and negative electron pairs in the quartz produce gamma photons after annihilation. which interact with $\mathrm{NaI}$, then it generates energy deposition. It can be seen from the simulation results that $5 \mathrm{~mm}$ quartz glass slice has met the design requirements of the detector.

\subsection{Algorithm Design}

\subsubsection{Total Activity of $\beta$ Aerosol}

If the concentration of $\beta$ aerosol is $\mathrm{C}\left(\mathrm{Bq} / \mathrm{m}^{3}\right)$, the filtration efficiency of filter membrane for aerosol is $\xi$, the detection efficiency of detector for $\beta$ is $\varepsilon$, gas flow rate is $\mathrm{Q}\left(\mathrm{m}^{3} / \mathrm{s}\right)$, and the counting increment in the measurement period $\Delta T$ (default is 1s) is $\Delta N / \Delta T$.

$$
\begin{aligned}
& \qquad \frac{\Delta N}{\Delta T}=C \times Q \times \Delta T \times \xi \times \varepsilon \\
& \Rightarrow C=\frac{\Delta N}{Q * \Delta T^{2} * \xi * \varepsilon}\left(\mathrm{Bq} / \mathrm{m}^{3}\right) \\
& \text { If } \mathrm{C} \text { is in } \mu \mathrm{CI} / \mathrm{m}^{3}, \text { then: } \\
& C=\frac{\Delta N}{3.7 * 10^{4} * \Delta T^{2} * Q * \xi * \varepsilon}\left(\mu \mathrm{Ci} / \mathrm{m}^{3}\right)
\end{aligned}
$$

\subsubsection{Minimum Detectable Activity Concentration (MDC)}

The MDC is as follows:

$$
\mathrm{MDC}=\frac{\mathrm{MDA}}{V}=\frac{2.71+4.65 \sqrt{N_{b}}}{Q * T * \xi * \varepsilon}
$$

In the formula, $\mathrm{N}_{\mathrm{b}}$ is the background $\beta$ count. $\mathrm{T}$ is the total measurement time in seconds. $Q$ is the gas flow rate in $\mathrm{m}^{3} / \mathrm{s}$, MDA is the Minimum Detectable Activity ${ }^{[6]}$

\section{CONCLUSION}

In this paper, based on the monitoring of radioactive aerosol from the stack of plasma melting system, there is no aerosol monitor in the market which can directly meet the requirements of high temperature environment. Combined with the source term analysis and measurement requirements of stack gas, the response curves of $\beta$ particles and $\gamma$ photons in plastic scintillator are simulated by Monte Carlo method, and the optimal scheme of detector design is analysed. A new detection design scheme using $0.25 \mathrm{~mm}$ plastic scintillator, $5 \mathrm{~mm}$ quartz glass and high temperature photomultiplier tube is adopted. The technology is mature, the cost is relatively low and the design of the detector and scintillator is simple and reliable. At present, the design is the first application at home, and the product have a wide range of applications. It provides an important data for the environmental protection department to evaluate the impact of stack gaseous effluent on the environment, and ensures a good health environment for local residents.

\section{References}

1. Chen Ming-zhou, Lv Yong-hong, etc. Advances in Research on Thermal Plasma Treatment of Low Intermediate Level Solid Radwastes from Npps[J], Radiation Protection, 2012,32(No.1):40-47.

2. Li Ai-wu, Zhang Zhi-long etc. A High Sensitive Radioactive Aerosol Continuous Monitor[J], Nuclear Electronics \& Detection Technology, 
2001,21(No.5):356-361.

3. Wang Yi-yuan, Wei Yong-kang, etc. Designing of Radioactive Aerosol Monitoring System for Nuclear Power Plant Based on PIPS Semiconductor Detector[J], Ship Science and Technology, 2011,33(No.8):137-139.

4. Lingqiu, Guo Lanying, Li Dongyu, etc. Radiometric measurement technology for nuclear power plants[M], China Beijing: Atomic Energy Press, 1998:162-167.

5. Li Xiang-dong, Gong Xue-yu, etc. PlasticNaI(TI)Crystal Composite Virtual Calibration Method of Detection Efficiency[J], University of South China(Science and Technology), 2011,25(No. 1):10-13.

6. Guo Xiao-bin, $\mathrm{Qu}$ Guo-pu, etc. Discuss the Technology for Dcrease the Detection Limit of $\mathrm{NaI}(\mathrm{TI})$ Gamma Spectrometer[J], University of South China(Science and Technology), 2011,25(No. 1):6-9. 